\title{
Current status and analysis of brain death organ donor's management through introduction of electronic notification system potential brain death donor
}

\author{
So Young Jeon ${ }^{1}$, Na Young Jeong ${ }^{1}$, Sun Mi Joํ․ Sang Heon Song ${ }^{2}$, Hyuk Jae Jung ${ }^{3}$, Jae Jun Jang ${ }^{4}$, Youn Jung Choi ${ }^{4}$, \\ Eun Jin Woo ${ }^{4}$
}

\footnotetext{
${ }^{1}$ Organ Transplantation Center, Pusan National University Hospital, Busan, Korea

${ }^{2}$ Department of Internal Medicine-Nephrology, Pusan National University Hospital, Busan, Korea

${ }^{3}$ Department of Surgery-Transplantation, Pusan National University Hospital, Busan, Korea

${ }^{4}$ Department of Yeongnam Management, Korea Organ Donation Agency, Busan, Korea
}

Background: According to the Act on Transplantation of Organs, etc., the head of a medical institution that has treated a potential brain death donor is required to notify the head of the organ procurement institution. Pusan National University Hospital has a potential brain death donor inquiry system, but there is a problem in that it does not have an automatic notification function. So, there is a problem in that the notification of the potential brain death donor is omitted by the medical staff due to busy, or the notification of the potential brain death donor is omitted when it is outside of regular hours. Restrictions on access are increasing due to the strengthening of intensive care unit (ICU) visitor records and access management, and visits by the Korea Organ Donation Agency (KODA) coordinator to discover potential brain death donor are restricted due to infection problems such as COVID-19. Recognizing the need for an untact way for discovering and notifying potential brain death donor while minimizing the risk of infection, a new electronic notification system for potential brain death donor was introduced, and I would like to introduce the current situation.

Methods: A computer was developed to generate a pop-up window when the condition that potential brain death donor criterion lasts for more than 6 hours is satisfied through the information entered in the clinical record of the ICU. Text message are sent to the KODA call center and the Pusan National University Hospital Organ Transplant Center's cell phone on duty. The new electronic notification systems for potential brain death donor operate from February 22, 2021. (Potential brain death donor criterion: loss of consciousness, coma; light reflex, fixed; pupil size, $4 \mathrm{~mm}$ or more; ventilator mode, CMV mode).

Results: Compared with the same period in 2020, the number of notification of potential brain death donors from March to June 2021 was the same at 30. However, it can remind medical staff of potential brain death donor notification system, and the notification process is simple as it is a one-click process. And it helps to make quick decisions, increasing work efficiency and simplifying the overall work flow.

Conclusions: Although the operation period is relatively short, there is a limit to analyze the effect of the application of the electronic notification system for potential brain death donor, but the improvement of work efficiency is considered an advantage. As it is an early stage using the potential brain death donor management system, it is being operated only for patients in neurosurgery, trauma surgery, and emergency medicine, but it is planned to be applied to all clinical departments in the future. In the future, it is thought that it is necessary to study the effect of the newly applied electronic notification system for potential brain death donor from a long-term perspective.

Corresponding author: So Young Jeon

E-mail: wjsth64@naver.com

(c) The Korean Society for Transplantation

This is an Open Access article distributed under the terms of the Creative Commons Attribution Non-Commercial License (http://creativecommons.org/licenses/by-nc/4.0/) which permits unrestricted non-commercial use, distribution, and reproduction in any medium, provided the original work is properly cited. 